\title{
Alternative Dark Energy Models: An Overview
}

\author{
J. A. S. Lima \\ Departamento de Física, Universidade Federal do Rio Grande do Norte, C.P. 1641, 59072-970, Natal, RN, Brasil
}

Received on 31 August, 2003.

\begin{abstract}
A large number of recent observational data strongly suggest that we live in a flat, accelerating Universe composed of $\sim 1 / 3$ of matter (baryonic + dark) and $\sim 2 / 3$ of an exotic component with large negative pressure, usually named Dark Energy or Quintessence. The basic set of experiments includes: observations from $\mathrm{SNe}$ Ia, CMB anisotropies, large scale structure, X-ray data from galaxy clusters, age estimates of globular clusters and old high redshift galaxies (OHRG's). It is now widely believed that such results provide the remaining piece of information connecting the inflationary flatness prediction $\left(\Omega_{\mathrm{T}}=1\right)$ with astronomical observations. From a theoretical viewpoint, they have also stimulated the current interest for more general models containing an extra component describing this unknown dark energy, and simultaneously accounting for the present accelerating stage of the Universe. In this review we present a simplified picture of the main results and discuss briefly some difficulties underlying the emerging dark energy paradigm.
\end{abstract}

\section{Introduction}

In 1998, some results based on Supernovae (SNe) type Ia observations published independently by two different groups, drastically changed our view about the present state of the universe $[1,2]$. In brief, the Hubble-Sandage diagram describing the observed brightness of these objects as a function of the redshift lead to unexpected and landmark conclusion: the expansion of the Universe is speeding up not slowing down as believed during many decades. Implicitly, such SNe type Ia observations suggest that the bulk of the energy density in the Universe is repulsive and appears like a dark energy component; an unknown form of energy with negative pressure [in addition to the ordinary dark matter] which is probably of primordial origin. In a more historical perspective, as the one shown in the chronological scheme below, one may say that contemporary cosmology started with the $\mathrm{SNe}$ "experiments". The current expectation is that important clues to the emerging dark energy paradigm will be provided by the next generation of SNe projects with advancing technology [3], as well as by a large set of complementary cosmological observations.

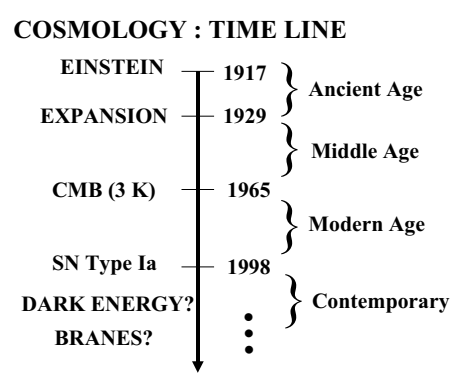

The existence of an extra component filling the Universe has also indirectly been suggested by independent studies based on fluctuations of the $3 \mathrm{~K}$ relic radiation [4], large scale structure [5], age estimates of globular clusters or old high redshift objects [6], as well as by the X-ray data from galaxy clusters [7]. Actually, the angular power spectrum of fluctuations in the cosmic microwave background (CMB) favors a model with total density parameter $\Omega_{T}=1$, a value originally predicted by inflation, whereas the density parameter associated with cold dark matter (CDM) is $\Omega_{m} \sim 0.3$, a value independently required by the power spectrum of the large scale structure (LSS) and X-ray data from galaxy clusters (see scheme above). The difference $\Omega_{D E}=\Omega_{T}-\Omega_{m} \sim 0.7$ is the density parameter of the dark energy component. Such a picture has recently been confirmed with even more precision by the Wilkinson Microwave Anisotropy Probe [8], and all these ingredients together reinforce what is usually referred to as the standard concordance model of cosmology [9].

\section{DARK ENERGY : GENEALOGY}

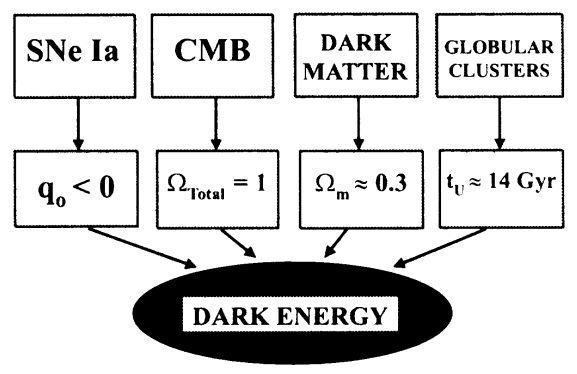

Although considering that dark energy changed the traditional view of the Universe, the absence of natural guidance from particle physics theory about its nature gave origin to an intense debate, as well as to many theoretical speculations. In particular, a cosmological constant $(\Lambda)-$ the oldest and by far the most natural candidate - is the simplest from a mathematical viewpoint but not the unique pos- 
sibility. The $\Lambda$ term was originally introduced by Einstein in 1917 to obtain a static world model. It is a time independent and spatially uniform dark component, which may classically be interpreted as a relativistic perfect simple fluid obeying the equation of state $p_{v}=-\rho_{v}$. In the framework of quantum field theory the presence of $\Lambda$ is due to the zeropoint energy of all particles and fields filling the Universe which manifests itself in several quantum phenomena like the Lamb shift and Casimir effect [10]. However, there is a fundamental problem related to such a theoretically favored candidate which is usually called the cosmological constant problem. Shortly, it is puzzling that the present cosmological upper bound $\left(\Lambda_{o} / 8 \pi G \sim 10^{-47} \mathrm{GeV}^{4}\right)$ differs from natural theoretical expectations $\left(\sim 10^{71} \mathrm{GeV}^{4}\right)$ by more than 100 orders of magnitude. This puzzle at the interface of astrophysics, cosmology, and quantum field theory has been considered by some authors as the greatest crisis of modern physics [11], and, as such, it acts like a Damocles sword on the cosmological constant solution for the present accelerating stage of the Universe.

Nowadays, there are many other candidates appearing in the literature, among them:

(i) a $\Lambda(t)$-term, or a decaying vacuum energy density.

(ii) a relic scalar field (SF) slowly rolling down its potential.

(iii) "X-matter", an extra component characterized by an equation of state $p_{\mathrm{x}}=\omega \rho_{\mathrm{x}},-1 \leq \omega<0$.

(iv) a Chaplygin-type gas whose equation of state is given by $p=-A / \rho^{\alpha}$, where $A$ is a positive constant and $0 \leq \alpha \leq 1$.

The list is by no means as exhaustive as one may think at first sight. Since the basic condition for an accelerating Universe is a dominant component with negative pressure, there are other possibilities which have occasionally been considered in the literature [12]. Note also that the model dominated by cosmological constant $\left(p_{v}=-\rho_{v}\right)$ is a limiting case of the X-matter parametrization $(\omega=-1)$.

The last three candidates above (SF, X-matter, and Chaplygin gas) share an additional physical property, namely, the effective equation of state parameter $(\omega(z)=p / \rho)$ may be a function of the redshift. In particular, this means that many different models may explain the same set of data. Therefore, in order to improve our understanding of the nature of dark energy, an important task nowadays in cosmology is to find new methods or to revive old ones that could directly or indirectly quantify the amount of dark energy present in the Universe, as well as determine its effective equation of state parameter. In other words, by learning more about the cosmic acceleration at low and high redshifts, one may expect to discriminate among the existing theories of dark energy by better determining $\omega$ and its time dependence.

In this short review we present a simplified picture of the main results and discuss briefly some difficulties of the emerging dark energy paradigm. Since the consequences of a cosmological constant and a rolling scalar field (usually considered the best candidates) have already been extensively discussed in recent review papers [13], in the present work we emphasize only the main results related to the remaining dark energy candidates.

\section{Alternative Dark Energy Models}

In what follows we restrict our attention to the class of spacetimes described by the FRW flat line element $(c=1)$

$$
d s^{2}=d t^{2}-R^{2}(t)\left[d r^{2}+r^{2}\left(d \theta^{2}+\sin ^{2} \theta \mathrm{d} \phi^{2}\right)\right],
$$

where $R(t)$ is the scale factor. Such a background is the spacetime favored by the cosmic concordance model since it is a direct consequence of the recent CMB results $\left(\Omega_{T}=1\right)$. Now, let us discuss the cosmic dynamics and some observational consequences for alternative dark energy candidates.

\section{A. Time-varying $\Lambda(t)$-term}

Decaying vacuum cosmologies or $\Lambda(t)$ models [14-20] are described in terms of a two-fluid mixture: a decaying vacuum medium $\left(\rho_{v}(t)=\Lambda(t) / 8 \pi G, p_{v}=-\rho_{v}\right)$ plus a fluid component ("decaying vacuum products") which are characterized by their energy density $\rho$ and pressure $p$. Historically, the idea of a time varying $\Lambda(t)$-term was first advanced in the paper of Bronstein [14]. Different from Einstein's cosmological constant, such a possibility somewhat missed in the literature for many decades, and, probably, it was not important to the recent development initiated by Ozer and Taha at the late eighties [15].

The Einstein field equations (EFE) and the energy conservation law (ECL) for $\Lambda(t)$ models are:

$$
\begin{gathered}
8 \pi G \rho+\Lambda(t)=3 \frac{\dot{R}^{2}}{R^{2}}, \\
8 \pi G p-\Lambda(t)=-2 \frac{\ddot{R}}{R}-\frac{\dot{R}^{2}}{R^{2}}, \\
\dot{\rho}+3 H(\rho+p)=-\frac{\dot{\Lambda}(t)}{8 \pi G},
\end{gathered}
$$

where a dot means time derivative. It should be noticed that the ECL (4) may be rewritten to yield an expression for the rate of entropy production in this model as

$$
T \frac{d S}{d t}=-\frac{\dot{\Lambda} R^{3}}{8 \pi G},
$$

showing that $\Lambda$ must decrease in the course of time, while the energy is transferred from the decaying vacuum to the material component (for more details see [15, 20, 22]).

At this point, we stress the difference between models with cosmological constant and a decaying vacuum energy density. In the later case, it is usually argued that the vacuum energy density is a time-dependent quantity because of its coupling with the other matter fields of the Universe. By virtue of the expansion, one may suppose that the cosmological constant is relaxing to its natural value $(\Lambda=0)$. Broadly speaking, the main goal of such models is to determine how the energy that drove inflation at early stages, and accelerates the universe at present is related to the current small value of $\Lambda$. Sometimes the decaying vacuum energy density is assumed to be an explicit time decreasing function. However, in the majority of the papers, it depends only 
implicitly on the cosmological time through the scale factor $\left(\Lambda \sim R^{-2}\right)$ or the Hubble parameter $\left(\Lambda \sim H^{2}\right)$, or even a combination of them [16, 18, 19]. An extensive list of phenomenological $\Lambda$-decay laws can be seen in the paper by Overduin and Cooperstock [21]. All these models have the same Achilles' heel: there is no Lagrangian description including the coupling term (nor any physical mechanism) governing the energy change between the decaying vacuum and other matter fields. The expression defining $\Lambda(t)$ is obtained either using dimensional arguments or in a completely ad hoc way. However, although essentially phenomenological, such an approach may indicate promising ways to solve the cosmological constant problem by establishing the effective regime to be provided by fundamental physics.

Certainly, one of the simplest possibilities for a decaying vacuum energy density is $\rho_{v}=\Lambda(t) / 8 \pi G=\beta \rho_{T}$, where $\rho_{v}$ is the vacuum energy density, $\rho_{T}=\rho_{v}+\rho$ is the total energy density, and $\beta \in[0,1]$ is a dimensionless parameter of order unity $[16,18]$. By combining such a condition with the first EFE equation one obtains the scaling law $\Lambda(t) \sim H^{2}$, a natural result from dimensional arguments. In this scenario, the expansion may be accelerated as required by SNe observations, and unlike the model proposed by Ozer and Taha [15] and Chen \& Wu [17] for which $\Lambda \sim R^{-2}$, it solves the age problem at $z=0$ [18].

From the observational viewpoint, $\Lambda(t) \mathrm{CDM}$ models possess an interesting characteristic that may distinguish them from $\Lambda \mathrm{CDM}$ models. Due to the possibility of an adiabatic photon production the standard temperature - redshift relation may be slightly modified. For a large class of models the temperature is given by $[20,22]$

$$
T(z)=T_{o}(1+z)^{1-\beta}
$$

where $T_{o}$ is the temperature of CMB at $z=0$. This expression implies that for a given redshift $z$, the temperature of the Universe is lower than in the standard photonconserved scenario. Although some recent determinations of $T(z)$ (based upon the $J=0,1$, and 2 ground state finestructure levels of $\mathrm{CI}$ ) have obtained values roughly consistent with the standard prediction, it is well known that such measurements must be taken as upper limits once many other excitations mechanisms may have contributed to the observed level populations. In particular, by considering collisional excitations, Molaro et al. [23] found a temperature for the CMB of $T_{\mathrm{CMB}}=12.1_{-3.2}^{+1.7} \mathrm{~K}$ at $z=3.025$. This result implies $\beta \leq 0.22$ at $2 \sigma$. More stringent constraints are furnished by big bang nucleosynthesis (BBN). Initially, Birkel and Sarkar [24] obtained $\beta \leq 0.13$, whereas a slightly greater upper bound, $\beta \leq 0.16$, was further derived by Lima et al. [25]. In such analyses, it was assumed that the $\beta$ parameter has the same value during the vacuumradiation and vacuum-matter dominated epochs. Probably, if one relaxes this hypothesis it will be much easier to satisfy the nucleosynthesis constraints and solve other cosmological problems. Constraints from SNe observations, angular diameter versus redshift

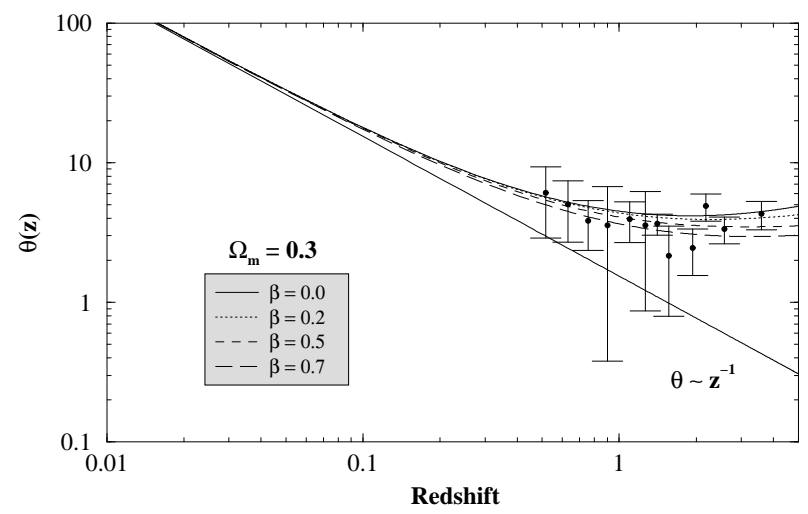

Figure 1. The angular size - redshift relation in decaying $\Lambda(t)$ models. The data set is composed by 145 milliarcsecond radio-sources distributed over a wide range of redshifts $(0.011 \leq z \leq 4.72)$ and binned into 12 bins [41]. The curves correspond to $\Omega_{\mathrm{m}}=0.3$ and a proper length $l=26.46 h^{-1} \mathrm{pc}$ (see [28] for more details).

relation, gravitational lensing and other kinematic tests (assuming a constant $\beta$ parameter) have been discussed by many authors [18, 19, 26, 27, 28, 29].

\section{B. X-Matter}

In the Cosmological scenarios driven by X-matter plus cold dark matter (sometimes called XCDM parametrization) both fluid components are separately conserved [30]. The equation of state of the dark energy component is $p_{x}=w(z) \rho_{x}$. Unlike to what happens with scalar field motivated models where $w(z)$ is derived from the field description [31], the expression of $w(z)$ for XCDM scenarios must be assumed a priori. Usually, it varies with some power of the redshift, say, $w(z)=w_{o}(1+z)^{n}$. Models with constant $w$ are the simplest ones and their free parameters can easily be constrained from the main cosmological tests.

More recently, in order to detect the possibility of bias in the parameter determination due to the imposition $\omega \geq-1$, some authors have studied models with constant $w$ by considering two different cases: the standard XCDM $(-1 \leq$ $\omega<0$ ) and the extended XCDM (also named "phantom" energy [32]) in which the $\omega$ parameter violates the null energy condition and may assume values $<-1$. In the case of $\mathrm{X}$-ray data from galaxy clusters, for instance, a good agreement between theory and observations for $w>-1$ is possible if $0.29 \leq \Omega_{\mathrm{m}} \leq 0.33$ (68.3\% c.1.) and $\omega \leq-0.55$ [33]. These results are in line with recent analyses from distant SNe Ia [34], SNe + CMB [35], Sne + LSS [36], gravitational lensing statistics [37] and the existence of old high redshift objects (OHRO's) [38]. In particular, Garnavich et al. [34] used the SNe Ia data from the High-Z Supernova Search Team to find $\omega<-0.55$ (95\% c.l.) for flat models whatever the value of $\Omega_{\mathrm{m}}$ whereas for arbitrary geometries they obtained $\omega<-0.6$ (95\% c.l.). Such results agree with the constraints obtained from a wide variety of different phenomena, using the "concordance cosmic" method [39]. In this case, the combined maximum likelihood analysis suggests $\omega \leq-0.6$, which ruled out dark components like topological defects (domain walls and string) for which $\omega=-n / 3$, 
being $n$ the dimension of the defect. More recently, Lima and Alcaniz [40] investigated the angular size - redshift diagram $(\theta(z))$ models by using the Gurvits' et al. published data set [41]. Their analysis suggests $-1 \leq \omega \leq-0.5$ whereas Corasaniti and Copeland [42] found, by using $\mathrm{SNe}$ Ia data and measurements of the position of the acoustic peaks in the CMB spectrum, $-1 \leq \omega \leq-0.93$ at $2 \sigma$. Jain et al. [43] used image separation distribution function $(\Delta \theta)$ of lensed quasars to obtain $-0.75 \leq \omega \leq-0.42$, for the observed range of $\Omega_{m} \sim 0.2-0.4$ while Chae et al. [37] used gravitational lens (GL) statistics based on the final Cosmic Lens All Sky Survey (CLASS) data to find $\omega<-0.55_{-0.11}^{+0.18}$ (68\% c.1.). Bean and Melchiorri [44] obtained $\omega<-0.85$ from $\mathrm{CMB}+\mathrm{SNe}$ Ia + LSS data, which provides no significant evidence for XCDM behaviour different from that of a cosmological constant. A similar conclusion was also obtained by Schuecker et al. [45] from an analysis involving the REFLEX X-ray cluster and SNe Ia data in which the condition $\omega \geq-1$ was relaxed.

The case for extended XCDM is an interesting one. First, it was observed that a dark component with $\omega<-1$ may provide a better fit to SNe Ia observations than do $\Lambda \mathrm{CDM}$ scenarios [32]. Although having some unusual properties, this "phantom" behavior is predicted by different approaches as, for example, kinetically driven models [48] and some versions of brane world cosmologies [49] (see also [50] and references therein). In actual fact, the best-fit model is considerably modified when the "phantom" behavior is allowed. In particular, for the X-ray data from galaxy cluster quoted above, it occurs for $\Omega_{\mathrm{m}}=0.31, \omega=-1.32$ and $\chi_{\min }^{2}=1.78$ [33]. Such limits are more restrictive than the ones obtained by Hannestad \& Mörtsell [51] by combining CMB + Large Scale Structure (LSS) + SNe Ia data. At $95.4 \%$ c.l. they found $-2.68<\omega<-0.78$.

A summary of recent constraints on the dark energy parameter $\omega$ is presented in Table I. As one may see there, the estimates of $\Omega_{\mathrm{m}}$ and $\omega$ are compatible with the results obtained from many independent methods. In general, joint analyses involving X-ray data, gravitational lensing, OHRG's, SNe type Ia, CMB, and other different methods are very welcome. First, in virtue of the gain in precision as compared to studies using only a specific set of data. The second reason, and, perhaps more important, is that most of cosmological tests are highly degenerate, thereby constraining only certain combinations of cosmological parameters but not each parameter individually.

\section{Chaplygin-type gas}

It is widely known that the main distinction between the pressureless CDM and dark energy is that the former agglomerates at small scales whereas the dark energy is a smooth component. Such properties seems to be directly linked to the equation of state of both components. Recently, the idea of a unified description for CDM and dark energy scenarios has received much attention. For example, Wetterich [52] suggested that dark matter might consist of quintessence

Table I. Limits to $\Omega_{\mathrm{m}}$ and $\omega$

\begin{tabular}{|c|c|c|c|}
\hline Method & Reference & $\Omega_{\mathrm{m}}$ & $\omega$ \\
\hline \multirow{2}{*}{$\mathrm{CMB}+$ SNe Ia:..... } & {$[30]$} & $\simeq 0.3$ & $\simeq-0.6$ \\
\hline & [35] & $\sim$ & $<-0.6$ \\
\hline $\mathrm{SNe} \mathrm{Ia}+\mathrm{LSS} \ldots .$. & [36] & $\sim$ & $<-0.6$ \\
\hline 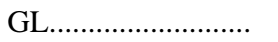 & [37] & $\sim$ & -0.55 \\
\hline X-ray GC...................... & [33] & $\simeq 0.32$ & -1 \\
\hline X-ray GC ${ }^{a} \ldots \ldots \ldots \ldots . . . . . . .$. & [33] & $\simeq 0.31$ & -1.32 \\
\hline 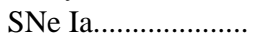 & [34] & $\sim$ & $<-0.55$ \\
\hline $\mathrm{SNe}+\mathrm{X}$-ray $\mathrm{GC}^{a}$. & [45] & $\simeq 0.29$ & $-0.95_{-0.35}^{+0.30}$ \\
\hline $\mathrm{SNe} \mathrm{Ia}+\mathrm{GL} \ldots \ldots \ldots . .$. & [46] & 0.24 & $<-0.7$ \\
\hline OHRO's.................... & [38] & 0.3 & $\leq-0.27$ \\
\hline 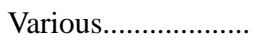 & [39] & $0.2-0.5$ & $<-0.6$ \\
\hline \multirow{2}{*}{$\theta(z) \ldots \ldots \ldots \ldots \ldots \ldots$} & [40] & 0.2 & $\simeq-1.0$ \\
\hline & [42] & $\sim$ & $<-0.96$ \\
\hline$\Delta \theta \ldots \ldots \ldots \ldots \ldots \ldots$ & [43] & $0.2-0.4$ & $\leq-0.5$ \\
\hline 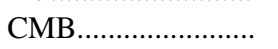 & [47] & 0.3 & $\overline{<}-0.5$ \\
\hline $\mathrm{CMB}+\mathrm{SNe}+\mathrm{LSS}$ & [44] & 0.3 & $<-0.85$ \\
\hline $\mathrm{CMB}+\mathrm{SNe}+\mathrm{LSS}$ & [51] & $\sim$ & $<-0.71$ \\
\hline $\mathrm{CMB}+\mathrm{SNe}+\mathrm{LSS}^{1}$ & [51] & $\sim$ & $>-2.68$ \\
\hline
\end{tabular}

lumps while Kasuya [53] showed that spintessence type scenarios are generally unstable to formation of $Q$ balls which behave as pressureless matter. More recently, Padmanabhan and Choudhury [54] investigated such a possibility trough a string theory motivated tachyonic field. Another interesting attempt at unification was suggested by Kamenshchik et al. [55] and further developed by Bilić et al. [56] and Bento et al. [57]. It refers to an exotic fluid, the so-called Chaplygin type gas $(\mathrm{Cg})$, whose equation of state is

$$
p_{C g}=-A / \rho^{\alpha}
$$

where $A$ and $\alpha=1$ are positive constants. The above equation for $\alpha \neq 1$ constitutes a generalization of the original Chaplygin gas equation of state proposed in Ref. [57] whereas for $\alpha=0$, the model behaves as $\Lambda$ CDM. The idea of a Unified Dark-Matter-Energy (UDME) scenario inspired by an equation of state like (7) comes from the fact that the Chaplygin type gas can naturally interpolate between non-relativistic matter and negative-pressure dark energy regimes $[56,57]$. Since in this approach there is only one dark component beside baryons, photons and neutrinos, some authors have termed this UDME scenario as a Quartessence cosmology [58].

Motivated by these possibilities, there has been growing interest in exploring the theoretical and observational consequences of the Chaplygin gas, not only as a possibility for unification of the dark sector (dark matter/dark energy) but also as a new candidate for dark energy only. The viability of such cosmological scenarios has been confronted by many observational results and their two free parameters have been constrained by many authors. For example, Fabris et al. [59] analyzed some consequences of such scenarios using type Ia supernovae data ( $\mathrm{SNe}$ Ia). Their results indicate that a cosmology completely dominated by 
the Chaplygin gas is favored in comparison to $\Lambda \mathrm{CDM}$ models. Recently, Avelino et al. [60] used a larger sample of SNe Ia and the shape of the matter power spectrum to show that such data restrict the model to a behaviour that closely matches that of a $\Lambda$ CDM models while Bento et al. $[61,62]$ showed that the location of the CMB peaks imposes tight constraints on the free parameters of the model. More recently, Dev, Alcaniz \& Jain [63] and Alcaniz, Jain \& Dev [64] investigated the constraints on the C-gas equation of state from strong lensing statistics and high- $z$ age estimates, respectively, while Silva \& Bertolami [65] studied the use of future SNAP data together with the result of searches for strong gravitational lenses in future large quasar surveys to constrain C-gas models. The trajectories of statefinder parameters [66] in this class of scenarios were studied in Ref. [67] while constraints involving Cosmic Microwave Background (CMB) data, Fanaroff-Ryley type IIb radio galaxies and X-ray data from galaxy clusters, have also been extensively discussed by many authors either as a dark energy or in the UDME picture [65, 62, 68, 69, 58].

\section{Conclusion}

The search for cosmologies driven by dark energy is presently in vogue. The leitmotiv is the observational support for an accelerated Universe provided by the type Ia supernovae $(\mathrm{SNe})$ experiments at intermediate and high redshifts.

This short review focused on some alternative candidates to dark energy. This ubiquitous component plus the dark matter are responsible for nearly $95 \%$ of the matterenergy content filling the Universe. However, different from dark matter, the extra dark (energy) component is intrinsically relativistic and its negative pressure is required by the present accelerating stage of the Universe. Its tiny density and weak interaction presumably preclude the possibility of identification in the terrestrial laboratory. Unfortunately, even considering that we are in the golden age of empirical cosmology, the existing data are still unable to discriminate among the different dark energy candidates, thereby signaling that we need better observations in order to test the basic predictions. In particular, this means that the determination of cosmological parameters will continue to be a central goal in the near future. The fundamental aim is to shed some light on the nature of the dark energy.

On the other hand, since the current models are more complicated than the Einstein-de Sitter Universe, such a situation is somewhat uncomfortable either from theoretical or observational viewpoints. It has also to be admitted that none dark energy model has been successful enough to deserve the status of a "standard model". However, the present time for many cosmologists is very exciting because although preserving some aspects of the basic physical picture, the new invisible actor (dark energy) which has not been predicted by particle physics, and is responsible for repulsive gravity, may alter profoundly the traditional view of space-time and matter.

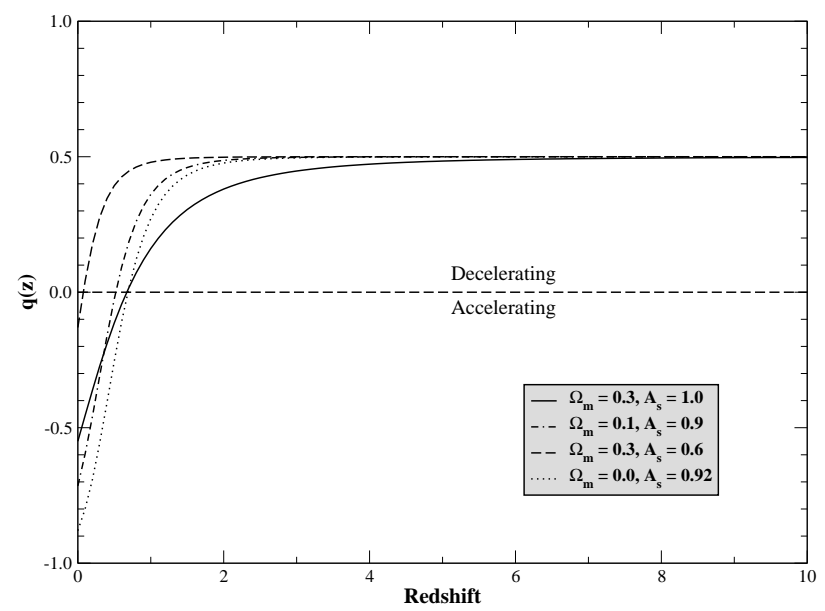

Figure 2. The Chaplygin gas solution for SNe observations. The plot shows the deceleration parameter in the original Chaplygin gas $(\alpha=1)$ as a function of redshift for some selected values of $\Omega_{\mathrm{m}}$ and $A_{s}=A \rho_{o}^{-2}$. The horizontal line $\left(q_{o}=0\right)$ divides models with a decelerating or accelerating expansion at a given redshift. Note that all models are accelerating at redshifts $z \lesssim 1$ (from Dev, Jain and Alcaniz [63]).

\section{Acknowledgments}

This work was partially supported by the Conselho Nacional de Desenvolvimento Científico e Tecnológico (CNPq), Pronex/FINEP (No. 41.96.0908.00), FAPESP (00/06695-0) and CNPq (62.0053/01-1-PADCT III/Milenio). I am indebted to G. Steigman, J. S. Alcaniz, N. Pires, R. Silva, J. V. Cunha and R. C. Santos for many helpful discussions.

\section{References}

[1] S. Perlmutter et al., Nature, 391, 51 (1998); S. Perlmutter et al., Astrop. J. 517, 565 (1999).

[2] A. Riess et al., Astron. J. 116, 1009 (1998).

[3] See the Web sites of the Supernova Cosmology Project (http://supernova.LBL.gov), and High-z Supernova Search (http://cfa-www.harvard.edu/supernova).

[4] P. de Bernardis et al., Nature 404, 955 (2000); L. Knox and L. Page, PRL 851366 (2000); A.H. Jaffe et al., PRL 86, 3475 (2001).

[5] R.G. Carlberg et al., Astrop. J. 462, 32 (1996); A. Dekel, D. Burstein and S.D.M. White, In Critical Dialogues in Cosmology, edited by N. Turok World Scientific, Singapore (1997); P. J. E. Peebles, in Formation of Structure in the Universe, edited by A. Dekel and J. P. Ostriker, Cambridge UP, Cambridge (1999).

[6] J. Dunlop et al., Nature 381, 581 (1996); Y. Yoshii, T. Tsujimoto and K. Kawara, ApJ 507, L133 (1998); J.S. Alcaniz and J.A.S. Lima, Astrop. J. 521, L87 (1999).

[7] G. Steigman and J.E. Felten, Space Sci. Rev. 74, 245 (1995); G. Steigman, N. Hata, and J.E. Felten, Astrop. J. 510, 564 
(1999); S.W. Allen, R. W. Schmidt, and A.C. Fabian, MNRAS 334, L11 (2002); S. Ettori, P. Tozzi, and P. Rosati, A\&A 398, 879 (2003).

[8] See the papers of the WMAP Collaboration: astro$\mathrm{ph} / 0302207-09,13-15,22-25$.

[9] J.P. Ostriker and P. Steinhardt, Science 300, 1909 (2003).

[10] M. Bordag, U. Mohideen, and V.M. Mostepanenko, Phys. Rep. 353, 1 (2001).

[11] Ya. B. Zeldovich, Sov. Phys. Usp. 11, 381 (1968); S. Weinberg, Rev. Mod. Phys. 61, 1 (1989).

[12] J.A.S. Lima and J.S. Alcaniz, A\&A 348, 1 (1999); L. P. Chimento, A. S. Jakubi, and N. A. Zuccala, Phys. Rev. D63, 103508 (2001); W. Zimdahl, D.J. Schwarz, A.B. Balakin, and D. Pavón, Phys. Rev. D64, 063501 (2001); M.P. Freaza, R.S. de Souza, and I. Waga, Phys. Rev. D66, 103502 (2002); K. Freese and M. Lewis, Phys. Lett. B 540, 1 (2002); Z. Zhu and M. Fujimoto, ApJ 581, 1 (2002).

[13] R. R. Caldwell, Braz. J. Phys. 30, 215 (2000); V. Sahni and A. Starobinsky, Int. J. Mod. Phys. D9, 373 (2000); T. Padmanabhan, Phys. Rep. 380, 235 (2003); P. J. E. Peebles and B. Ratra, Rev. Mod. Phys. 75, 559 (2003).

[14] M. Bronstein, Phys. Z. Sowjetunion 3 (1933).

[15] M. Ozer and M. O. Taha, Phys. Lett. B 171, 363 (1986); Nucl. Phys. B287 776 (1987).

[16] K. Freese, F.C. Adams, J.A. Frieman, and E. Mottola, Nucl. Phys. B287, 797 (1987); M. S. Berman, Phys. Rev. 43, 1075 (1991); D. Pavón, Phys. Rev. D43, 375 (1991); M.O. Calvão, H.P. de Oliveira, D. Pavón , J.M. Salim, Phys. Rev. D45, 3869 (1992).

[17] W. Chen and Y-S. Wu, Phys. Rev. D41, 695 (1990).

[18] J.C. Carvalho, J.A.S. Lima, and I. Waga, Phys. Rev. D46 2404 (1992).

[19] A.-M. M.Abdel-Rahman, Phys. Rev. D45, 3497 (1992); I. Waga, ApJ 414, 436 (1993); J. M. F. Maia and J.A.S. Lima, Mod. Phys. Lett. A8, 591 (1993); A. Beesham, Phys. Rev. D48, 3539 (1993); J. A.S. Lima and J.M.F. Maia, Phys. Rev. D49, 5597 (1994); A. I. Arbab, and A.M.M. Abdel-Rahman, Phys. Rev. D50, 7725 (1994); J. Matygasek, Phys. Rev. D51, 4154 (1994).

[20] J.A.S. Lima and M. Trodden, Phys. Rev. D53, 4280 (1996); J.A.S. Lima, Phys. Rev. D54, 2571 (1996).

[21] J. M. Overduin and F. I. Cooperstock, Phys. Rev. D58, 043506 (1998).

[22] J. A. S. Lima, A. I. Silva, and S. M. Viegas, MNRAS 312, 747 (2000).

[23] P. Molaro et al., A\&A 381, L64 (2002).

[24] M. Birkel and S. Sarkar, Astrop. Phys. 6, 197 (1997).

[25] J.A.S. Lima, J.M.F. Maia, and N. Pires, IAU Simposium 198, $111(2000)$

[26] L. F. Bloomfield Torres and I. Waga, MNRAS 279, 712 (1996).

[27] R. G. Vishwakarma, CQG 17, 3833 (2000); CQG 18, 1159 (2001).

[28] J. V. Cunha, J.A.S. Lima, and N. Pires, A\&A 390, 809 (2002); J. V. Cunha, J.A.S. Lima, and J. S. Alcaniz, Phys. Rev. D66, 023520 (2002).
[29] J.S. Alcaniz and J.M.F. Maia, Phy. Rev. D67, 043502 (2003).

[30] M.S. Turner and M. White, Phys. Rev. D56, R4439 (1997); T. Chiba, N. Sugiyama, and T. Nakamura, Mon. Not. Roy. Astron. Soc. 289, L5 (1997); J. S. Alcaniz and J.A.S. Lima, Astrop. J. 550, L133 (2001); J. Kujat, A. M. Linn, R.J. Scherrer, and D.H. Weinberg, ApJ 572, 1 (2002).

[31] T.D. Saini, S. Raychaudhury, V. Sahni, and A.A. Starobinsky, Phys. Rev. Lett. 85, 1162 (2000); J.K. Erickson et al., Phys. Rev. Lett. 88121301 (2002).

[32] R.R. Caldwell, Phys. Lett. B545, 23 (2002).

[33] J.A.S. Lima and J. S. Alcaniz, MNRAS 317, 893 (2000); J. A. S. Lima, J. V. Cunha and J. S. Alcaniz, Phys. Rev. D 68, 023510 (2003).

[34] P.M. Garnavich et al., ApJ 509, 74 (1998)

[35] G. Efstathiou, MNRAS 310, 842 (1999).

[36] S. Perlmutter, M. S. Turner, and M. White, Phys. Rev. Lett. 83, 670 (1999).

[37] K.-H. Chae et al., Phys. Rev. Lett. 89, 151301 (2002).

[38] J.S. Alcaniz, J.A.S. Lima, and J. V. Cunha, MNRAS 340, L39 (2003).

[39] L.M. Wang, R.R. Caldwell, and J.P. Ostriker \& P. J. Steinhardt, Astrophys. 530, 17 (2000)

[40] J. A. S. Lima and J. S. Alcaniz, Astrophys. 566, 15 (2002).

[41] L.I. Gurvits, K.I. Kellermann, and S. Frey, A\&A 342, 378 (1999).

[42] P.S. Corasaniti and E.J. Copeland, Phys. Rev. D65, 043004 (2002).

[43] D. Jain, A. Dev, N. Panchapakesan, S. Mahajan, and V.B. Bhatia, Int. J. Mod. Phys. D12, 953 (2003).

[44] R. Bean and A. Melchiorri, Phys. Rev. D65, 041302 (2002).

[45] P. Schuecker, R. R. Caldwell, H. Bohringer, C. A. Collins, and Luigi Guzzo, A\&A 402, 53 (2003).

[46] I. Waga and A.P.M.R. Miceli, Phys. Rev 59, 103507 (1999).

[47] A. Balbi, C. Baccigalupi, S. Matarrese, F. Perrota, and N. Vittorio, Astrop. J. 547, L89 (2001).

[48] T. Chiba, T. Okabe, and M. Yamaguchi, Phys. Rev. D62, 023511 (2000).

[49] V. Sahni and Y. Shtanov, "Brane World Models of Dark Energy”, astro-ph/0202346.

[50] B. McInnes, in "On the Nature of Dark Energy", edited by P. Brax, J. Martin, J.-P. Vzan, Proceeding of the XVIII th Coloquium, Paris (2002). astro-ph/0210321; S.M. Carroll, M. Hoffman, and M. Trodden, Phys. Rev. D68, 023509 (2003).

[51] S. Hannestad and E. Mörtsell, Phys. Rev D66, 063508 (2002).

[52] C. Wetterich, Phys. Rev. D65, 123512 (2002).

[53] S. Kasuya, Phys. Lett. B515, 121 (2001).

[54] T. Padmanabhan and T. R. Choudhury, Phys. Rev. D66, 081301 (2002).

[55] A. Kamenshchik, U. Moschella, and V. Pasquier, Phys. Lett. B511, 265 (2001). 
[56] N. Bilic, G.B. Tupper, and R.D. Violler, Phys. Lett. B535, 17 (2002).

[57] M.C. Bento, O. Bertolami and A.A. Sen, Phys. Rev. D66, 043507 (2002).

[58] M. Makler, S.Q. de Oliveira, and I. Waga, Phys. Lett. B555, 1 (2003).

[59] J.C. Fabris, S.V.B. Gonçalves, and P. E. de Souza, astro$\mathrm{ph} / 0207430$

[60] P.P. Avelino, L.M.G. Beça, J.P.M. de Carvalho, C.J.A.P. Martins, and P. Pinto, Phys. Rev. D 67, 023511 (2003).

[61] M.C. Bento, O. Bertolami, and A.A. Sen, Phys. Rev. D67, 063003 (2003).

[62] M.C. Bento, O. Bertolami, and A.A. Sen, astro-ph/0303538; gr-qc/0305086.

[63] A. Dev, J.S. Alcaniz, and D. Jain, Phys. Rev D 67, 023515 (2003). See also astro-ph/0209379.
[64] J.S. Alcaniz, D. Jain, and A. Dev, Phys. Rev. D67, 043514 (2003). See also astro-ph/0210476.

[65] P.T. Silva and O. Bertolami, astro-ph/0303353.

[66] V. Sahni, T.D. Saini, A.A. Starobinsky, and U. Alam, JETP Lett. 77, 201 (2003).

[67] V. Gorini, A. Kamenshchik, and U. Moschella, Phys. Rev. D 67, 063509 (2003); U. Alam, V. Sahni, T.D. Saini, and A.A. Starobinsky, astro-ph/0303009.

[68] D. Carturan and F. Finelli, astro-ph/0211626; L. Amendola, F. Finelli, C. Burigana, and D. Carturan, JCAP 0307, 005 (2003).

[69] J.V. Cunha, J.S. Alcaniz, and J.A.S. Lima, Phys. Rev D , (2003), in press. See also astro-ph/0306319.

[70] M. Makler, S.Q. de Oliveira and I. Waga, astro-ph/0306507. 